\title{
Miranda
}

Revue pluridisciplinaire du monde anglophone /

Multidisciplinary peer-reviewed journal on the English-

speaking world

23 | 2021

Modernist Exceptions

\section{Rap et fiction : Exploiter le flow pour traduire le roman urbain}

Tiffane Levick

\section{OpenEdition}

Journals

Édition électronique

URL : https://journals.openedition.org/miranda/41004

DOI : 10.4000/miranda.41004

ISSN : 2108-6559

Éditeur

Université Toulouse - Jean Jaurès

\section{Référence électronique}

Tiffane Levick, « Rap et fiction : Exploiter le flow pour traduire le roman urbain », Miranda [En ligne], 23 |

2021, mis en ligne le 04 octobre 2021, consulté le 29 novembre 2021. URL : http://

journals.openedition.org/miranda/41004; DOI : https://doi.org/10.4000/miranda.41004

Ce document a été généré automatiquement le 29 novembre 2021.

\section{(c) (i) (5)}

Miranda is licensed under a Creative Commons Attribution-NonCommercial-NoDerivatives 4.0 International License. 


\title{
Rap et fiction : Exploiter le flow pour traduire le roman urbain
}

\author{
Tiffane Levick
}

1 Cet article a pour but de mettre en évidence, dans une démarche à la fois réflexive et pratique, les différentes facettes d'une stratégie pour traduire le roman urbain qui s'appuie sur les techniques employées dans le rap. Par le biais d'exemples concrets tirés de mes traductions du français vers l'anglais, j'illustrerai les caractéristiques du rap que l'on peut exploiter pour faire valoir les fonctions créatives, ludiques, et rebelles de l'argot. Déployer cette stratégie dans la traduction de romans urbains permet notamment d'éviter le recours à des démarches telles la standardisation ou l'équivalence qui contribueraient à estomper ou à effacer les fonctions attribuées à la langue du texte original ou les spécificités de la voix des personnages.

2 Avant d'envisager les caractéristiques linguistiques et musicales des chansons de rap, il est utile de situer ce genre musical dans son contexte géographique, historique, et politique. Le rap voit le jour lors de la naissance du mouvement hip-hop dans les années 1970, dans le Bronx à New York, et constitue l'une des quatre branches du mouvement, aux côtés du DJing, du graffiti, et de la breakdance. Certains spécialistes en la matière soutiennent que l'ampleur du mouvement dépasse ces quatre branches, dont le DJ Kool Herc qui affirme qu'en plus de ces quatre pratiques artistiques, le hip-hop englobe également, entre autres, la démarche d'une personne, sa façon de s'exprimer, et son allure ${ }^{1}$ (Chang 2005, xii).

3 Le mouvement s'est concrétisé dans un contexte de pauvreté, d'exclusion et de chômage, constituant ainsi une manifestation de l'ennui et de la frustration des jeunes habitants des quartiers laissés à l'abandon. Les formes artistiques du mouvement permettent à cette jeunesse de manifester, de façon publique, leur existence et leur place dans la société à travers une performance contre l'invisibilité. En effet, selon Laurent Mucchielli, les personnes qui pratiquent le rap «[...] se sentent non pas trahi[e]s mais radicalement inentendu[e]s par les institutions nationales censées ouvrir et protéger les mêmes droits pour chaque citoyen» $(1999,4)$. Le rap leur permet donc 
de s'exprimer librement, de mettre des mots sur leurs inquiétudes de manière créative, et de se faire entendre par un public plus large que celui de leurs environs immédiats.

Bien qu'officiellement inaugurées dans les années 1970, les pratiques musicales et orales qui ont façonné la forme du rap remontent à des époques bien plus anciennes et comprennent notamment le blues, le gospel, le funk, et le jazz. En effet, selon Adam Bradley, le rap est un genre musical de la nouvelle école et un genre poétique de l'ancienne école (2009, xiv), et fait ainsi appel à une vaste gamme de techniques exploitées dans d'autres genres. Les meilleurs textes de rap, selon Adam Bradley et Andrew DuBois, sont intéressants sur le plan poétique, par nécessité ; ils n'ont que peu de mélodie ou d'harmonie pour pallier la faiblesse des paroles (2010, xxxiv). Le son distinct d'une chanson de rap se fait entendre par le biais du flow, la cadence lyrique du rappeur, le plus souvent relative à un rythme: la rencontre du rythme et de la rime (Bradley, 2009, 6).

5 Pour essayer de surprendre les auditeurs et de dynamiser les paroles, les MC ont tendance à vouloir subvertir dans leurs paroles, comme dans leur performance, les attentes du public. Cette tentative de subversion ressort en particulier dans la recherche de formes créatives à l'intérieur des contraintes des temps et mesures des vers d'une chanson. On voit donc des schémas de rimes complexes, contenant des rimes parfaites et imparfaites, des rimes mono- ou multi-syllabiques, et des finales aussi bien qu'internes. Ce jeu de sonorités s'étend pour comprendre d'autres procédés tels l'homophonie et l'homonymie, l'allitération, l'assonance, et la consonance, de même que l'onomatopée et les répétitions internes. Les paroles de rap contiennent également de nombreuses figures de style et d'images, notamment des métaphores filées (Bradley, 2009). Tous ces traits stylistiques fournissent une base à partir de laquelle les MC peuvent produire une performance personnelle, les paroles proposant une certaine souplesse quand elles sont portées à la scène. En effet, malgré les contraintes mentionnées plus haut liées aux temps et aux mesures, un rappeur peut contourner en partie ces limites en accentuant ou en réduisant certaines syllabes pour produire l'effet désiré, tout en évitant les pauses gênantes, halètements, ou autres irrégularités (Bradley, 25 et 29).

\section{Qu'est-ce que l'argot?}

6 Avant de voir comment le rap peut être utile dans la traduction de romans urbains, et en particulier du style argotique présent dans ces œuvres, il est utile de s'attarder sur les formes linguistiques fréquemment utilisées dans ces textes, à savoir l'argot. Pour délimiter la signification du terme, on peut s'appuyer sur la définition en anglais qui est proposée pour slang dans Global English Slang: Methodologies and Perspectives, un livre dans lequel un certain nombre de spécialistes explorent les traits et l'usage de différents argots dans le monde. Selon ces spécialistes :

L'argot est un langage informel, non technique, qui, pour l'utilisateur et/ou l'auditeur, a souvent l'air novateur, et qui remet en cause une norme sociale ou linguistique. Il peut également suggérer une complicité dans les jugements de valeur et jouer ainsi un rôle performatif dans la définition d'une identité individuelle ou de groupe. (Coleman 2014, 30) ${ }^{2}$

Cette définition a le mérite d'établir un consensus sur les différentes nuances du concept sociolinguistique désigné par le terme slang, permettant ainsi de poursuivre la 
discussion en analysant plus précisément la manière dont les caractéristiques de l'argot donnent la possibilité aux jeunes personnes faisant partie de groupes marginalisés d'aborder, directement ou indirectement, les questions d'altérité, d'appartenance, et d'identité.

8 En outre, l'idée proposée dans cette définition selon laquelle l'argot remet en cause une norme sociale ou linguistique invite à une réflexion sur la manière dont l'argot peut être considéré comme une forme de langue non standard. La définition de langue standard étant épineuse, il convient d'affirmer que l'idée du standard indique la variété de la langue qui est pratiquée et approuvée par les groupes sociaux dominants et que, par extension, l'argot peut être utilisé comme un moyen par lequel les membres de groupes marginalisés cherchent à mettre en avant leur différence, consciemment ou inconsciemment, par rapport à ces groupes dominants qui décident des normes sociales et linguistiques et les imposent à l'ensemble de la société. Il s'agit donc d'une forme de lutte de pouvoir, similaire à celle que l'on observe dans le rap.

Le spécialiste Eric Partridge mentionne en particulier le côté ludique et créatif de l'argot, et explique que son usage peut aider à décontracter une situation tendue, communiquer une certaine complicité entre interlocuteurs et l'appartenance à un groupe distinct, et exclure des personnes qui ne maîtrisent pas cette variété de la langue (Partridge 1933, 5). L'argot implique également un certain sens de la compétition entre ses locuteurs qui cherchent souvent à s'emparer de ses traits les plus impressionnants pour intimider leurs pairs. De nombreux théoriciens soulignent la place du lexique dans la formation de l'argot, et certains sont de l'avis que l'argot se réduit à un lexique qu'un locuteur peut choisir d'insérer dans une syntaxe familière (voir en particulier Antoine 2004, Doran 2007, François-Geiger 1989, et Valdman 2000). Cependant, qu'elles relèvent de l'argot ou d'une façon de parler dite familière, un certain nombre de caractéristiques linguistiques sont fréquemment observables dans des phrases argotiques, dont la préfixation et la suffixation, la composition et l'amalgame, la troncation, et l'emprunt de mots étrangers (Antoine 2004, 17). En somme, il s'agit d'outils linguistiques qui correspondent aux différentes fonctions citées plus haut.

10 Le débat autour de la différence entre l'argot et la langue familière invite à une réflexion sur la différence entre un argot dit plus général, accessible à l'ensemble des membres d'une communauté linguistique, et un argot plus "codé» dont l'usage est réservé à un groupe particulier dans cette communauté. Selon cette différenciation, l'emploi d'un argot codé permet aux locuteurs d'afficher de façon plus évidente leur appartenance à un groupe précis. De ce fait, une personne qui essaie de s'exprimer en se servant des caractéristiques de l'argot codé d'un groupe dont elle ne fait pas partie fera face à des problèmes de crédibilité et cette question peut être liée à la reproduction écrite et à la traduction de l'argot dans des textes de fiction.

11 Quand un écrivain choisit d'inclure des caractéristiques d'un argot dans un texte de fiction, il est utile d'interroger sa maîtrise de l'argot en question. On peut également étudier la façon dont l'argot est inclus dans le texte, placé uniquement dans le dialogue ou utilisé également dans la narration. De manière générale, on peut dire qu'un auteur qui décide d'écrire un texte contenant de l'argot se sert des traits les plus identifiables et compréhensibles de cet argot pour permettre à ses lecteurs de le comprendre et de le reconnaître. Pour amplifier l'impression de l'authenticité et l'oralité de son texte, l'écrivain peut par ailleurs exploiter certaines formes linguistiques que l'on utilise lors 
de conversations informelles. L'argot fictionnel est donc souvent une forme adoucie de l'argot réel, qui correspond dans une certaine mesure aux normes stylistiques de la tradition littéraire en question, tout comme les paroles de rap, qui se dotent d'une identité propre au MC quand elles sont proférées lors d'une performance.

\section{Comment traduire l'argot dans un roman?}

12 Mes lectures de textes traductologiques ainsi que des versions traduites de plusieurs œuvres dont l'histoire se déroule en banlieue parisienne m'ont menée, dans ma thèse de doctorat, à identifier cinq stratégies principales utilisées dans la traduction de l'argot apparaissant dans une œuvre de fiction: la standardisation, l'équivalence, l'invention, l'emprunt, et, finalement, l'emploi d'un style familier dit neutre. Chacune de ces stratégies est appliquée de manière variable dans la traduction de romans urbains, et il est bien entendu peu judicieux de les voir comme des procédures entièrement distinctes car certaines caractéristiques de ces différentes stratégies peuvent se superposer. Il est tout de même utile de les présenter individuellement pour faciliter l'identification des techniques utilisées et les conséquences de leur usage sur, par exemple, l'accessibilité du texte traduit, et son authenticité supposée.

Dans le cas où le traducteur choisit de remplacer les caractéristiques de l'argot employé dans le texte source par des mots ou expressions relevant de la variété standard de la langue cible, on peut considérer que son approche est une forme de standardisation. Le traducteur aura peut-être recours à l'emploi de cette stratégie s'il considère que le fond du texte contient suffisamment d'informations contextuelles qui correspondent aux différentes fonctions de l'argot, notamment la rébellion et l'appartenance à un groupe marginalisé. On peut également considérer cette stratégie comme une façon de rendre le texte traduit plus compréhensible et attractive pour un public qui serait réticent à l'idée de se plonger dans la lecture d'un texte d'origine étrangère. Il n'en reste pas moins que l'emploi de cette stratégie a pour résultat d'affaiblir la force stylistique de l'œuvre et de la réduire à son simple intérêt sémantique.

Une deuxième stratégie consiste à utiliser un argot "équivalent ». Par équivalent, j'entends le remplacement de l'argot du texte source par un argot utilisé par un groupe comparable vivant dans un lieu précis où la langue cible est parlée. L'application de cette stratégie peut encourager le lecteur à mieux comprendre le contexte du texte original en créant des parallèles entre ce contexte étranger et un contexte local, mais il s'avère difficile de produire une version crédible de l'argot choisi. En outre, l'emploi d'un argot équivalent peut entraîner l'effacement de la signifiance du contexte original si cet argot suppose un contexte alternatif.

15 Certains traducteurs optent également pour l'invention d'une nouvelle façon de parler dans la langue cible pour éviter d'utiliser ces deux premiers procédés. Ce nouvel argot peut se limiter au lexique, avec des mots et expressions inventés qui sont insérés dans des phrases construites selon les règles grammaticales de la langue cible, ou bien prendre une autre forme plus radicale qui concerne toute la structure de la langue. Dans les deux cas, l'invention peut représenter une façon de reproduire les fonctions ludiques de l'argot mais implique un défi considérable de cohérence et risque de placer le texte dans un espace flou sans ancrage réel. la langue originale et transféré directement, avec ou sans modification orthographique, 
du texte source au texte cible, avec ou sans l'addition de mots ou d'explications qui faciliteraient la compréhension pour le lecteur. Cette stratégie souligne l'origine étrangère $\mathrm{du}$ texte mais peut également exoticiser le cadre original ou, sinon, réduire l'aspect de l'étranger à l'ensemble des locuteurs de la langue au lieu des membres du groupe qui utilisent l'argot en question, puisque le lecteur du texte traduit n'a sans doute pas les compétences linguistiques nécessaires pour distinguer la différence entre les variétés de la langue standard et non standard du contexte originel.

Une dernière approche consiste à employer une langue familière mais non argotique, qui évite donc de suggérer un cadre alternatif pour le texte. Cette approche est souvent privilégiée dans la traduction du dialogue, et peu de théoriciens ont développé une discussion poussée qui prenne en compte les implications de cette stratégie appliquée à un texte dans son intégralité. C'est cette stratégie qui a servi de point de départ à une sixième stratégie que j'ai développée dans le cadre de ma thèse, et que j'ai affinée depuis.

\section{Une sixième stratégie : «Le parler jeune global »}

18 Cette stratégie tente de compléter l'aspect familier du style en y ajoutant des traits qui relèvent plus spécifiquement d'un parler jeune, et de jeunes marginalisés, puisant dans techniques utilisées dans les paroles de rap. Cette fusion du familier et du rap me paraissait pertinente, en raison notamment des conditions similaires dans lesquelles l'argot et le rap sont utilisés; un contexte marqué par une tension sociale entre les groupes dominés et les groupes dominants poussent les membres de groupes marginalisés à vouloir s'exprimer par le biais de la performance verbale. Cette stratégie constitue donc une tentative de production d'un texte dont le style est relâché et oralisé, mais qui ne suggère pas de lieu précis.

Cette approche consiste à identifier par le biais de la traduction une sorte de "parler jeune global» en anglais, qui ne représente pas un effort d'inventer une sorte d'esperanto, mais qui au contraire exploite les traits les plus répandus des différentes variétés d'anglais utilisées par des jeunes anglophones dans divers pays, dans une situation sociale similaire. Mon hypothèse de départ était qu'il existait des caractéristiques de la langue anglaise qui étaient partagées par tous les jeunes anglophones du monde, quel que soit leur pays, et que ces caractéristiques devenaient de plus en plus omniprésentes dans la langue notamment grâce aux nouvelles technologies qui permettent davantage de communication entre les différentes cultures du monde, ainsi qu'en raison de la disponibilité de textes écrits et audiovisuels en provenance du monde entier. Une fois l'approche globale de la traduction établie, il a fallu mettre en place des techniques concrètes pour en assurer la réalisation.

Le fondement de cette démarche se manifeste dans l'emploi d'une syntaxe relâchée qui évite des mots, expressions, et tournures qui risquent de déplacer le texte dans un lieu précis du monde anglophone. Plus précisément, on peut avoir recours : à l'usage de contractions qui indiquent le débit de la parole (ex. "shouldn't've») mais qui ne suggèrent pas un accent particulier ; à des mots de remplissage (ex. « or anything »); à la suppression des pronoms personnels où c'est possible; enfin, à l'emploi de démonstratifs familiers. Tout cela doit être effectué en évitant des choix qui pourraient sembler trop typiques d'une communauté linguistique anglophone particulière. Les caractéristiques du rap viennent compléter ces traits du "parler jeune global», le flow 
étant un aspect primordial de la stratégie de traduction qui permet de proposer un texte traduit dont le style est à la fois ludique et créatif, en conformité avec les fonctions de l'argot. Pour illustrer cette démarche, je propose ci-dessous une série d'exemples de longueurs diverses tirés de passages narratifs de quatre romans que j'ai traduits, en entier ou en partie, depuis 2018: Moi non, le roman de Patrick Goujon publié par Gallimard en 2003 que j'ai traduit dans le cadre de ma thèse de doctorat ; Fief, de David Lopez, publié par les Éditions du Seuil en 2017 ; Paname Underground, de Zarca, publié par les Éditions Goutte d'Or en 2017 ; et Rhapsodie des oubliés, de Sofia Aouine, publié par les Éditions de la Martinière en 2019.

\section{Moi non}

\begin{tabular}{|l|l|}
\hline $\begin{array}{l}\text { Juste je suis assis là, à me répéter des } \\
\text { mensonges, à regarder ma chemise en soie } \\
\text { salopée par le cambouis du Tanplain }\end{array}$ & $\begin{array}{l}\text { So I just stay sitting where I am, silently } \\
\text { reciting the same old lies, looking at my silk } \\
\text { shirt, shit-soiled by the grease of Diorestore. }\end{array}$ \\
\hline $\begin{array}{l}\text { Dans mon attirail,j'ai aussi un masque pour la } \\
\text { poussière mais comment travailler avec un } \\
\text { bordel pareil sur le nez, qui filtre la crasse } \\
\text { mais vous rationne du même coup la } \\
\text { respiration? }\end{array}$ & $\begin{array}{l}\text { There's also a dust mask in amongst all my gear } \\
\text { but how am I supposed to work with a thing } \\
\text { but it sure stops you from breathing freely. }\end{array}$ \\
$\begin{array}{l}\text { Pour l'instant, c'est lits superposés pour les } \\
\text { familles nombreuses mais ça viendra, le } \\
\text { principe des étagères aux murs. }\end{array}$ & $\begin{array}{l}\text { For now, it's bunk beds for big families but the } \\
\text { shelves-on-walls concept will come. }\end{array}$ \\
\hline
\end{tabular}

21 Les trois exemples présentés ci-dessus illustrent la façon dont l'assonance et la consonance peuvent contribuer à instaurer un flow dans le texte traduit, les sons soulignés en gras étant repris plusieurs fois. On voit également ce travail sur le rythme à travers les terminaisons (ing) et dans les échos produits par les syllabes (bunk, beds, big ; silk, shirt, shit), ainsi que dans les deux mots composés (shit-soiled, shelves-on-walls) dont l'assemblage sert au cadencement des phrases.

Fief

C'est l'été que j'ai appris à traîner le soir. Une fois, Ixe est arrivé en disant hey les gars, vous savez quoi, j'ai du shit. Il avait pris ça dans une boîte qu'il y avait dans la chambre de son grand frère, et ça nous faisait un peu flipper, parce que son grand-frère c'était un mec pas très commode. Il ne traînait pas chez nous, on le connaissait à peine, il était surtout fourré avec les mecs des Tours. On avait peur qu'il se fasse griller Ixe, mais très vite on avait oublié tout ça et on s'apprêtait à fumer un joint pour la première fois ensemble.
It was during the summer that I learned to loiter at night. This one time, Ixe showed up saying Hey guys, guess what, I've got hash. He'd taken it from a box in his older brother's bedroom which kind of freaked us a out bit since his brother wasn't exactly the most upstanding of guys. He never spent any time in our neighbourhood and we barely knew him - he was always with the high-rise guys. We were scared that Ixe'd get caught, but forgot all that pretty quickly and got geared up to smoke our first joint together.

Fief, le deuxième roman dont j'ai traduit un chapitre à l'aide du parler jeune global, présente, comme Moi non, la voix d'une jeunesse masculine vivant en banlieue parisienne, cette fois-ci dans une zone dite périurbaine. Pour traduire l'argot des personnages et du jeune narrateur, j'ai procédé de la même manière, tentant de 
trouver des allitérations et des répétitions de sons (learned to loiter, got geared up) et d'insérer des mots composés (high-rise guys) dans des phrases remplies de groupes verbaux contractés (Ixe'd) et de tournures de remplissage (kind of), le tout afin de créer un style enjoué et rythmé.

\section{Paname Underground}

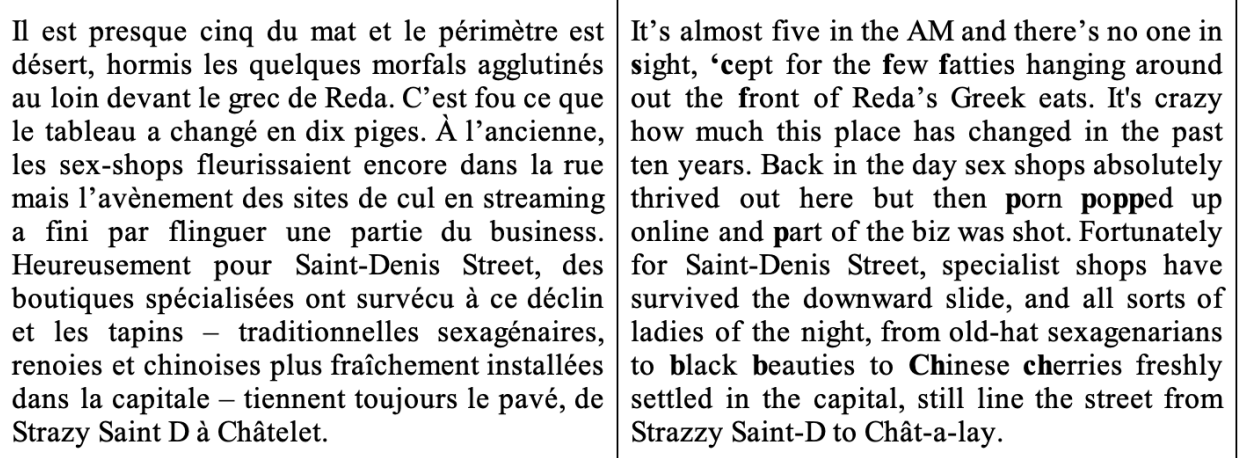

It's almost five in the AM and there's no one in sight, 'cept for the few fatties hanging around out the front of Reda's Greek eats. It's crazy how much this place has changed in the past ten years. Back in the day sex shops absolutely thrived out here but then porn popped up online and part of the biz was shot. Fortunately for Saint-Denis Street, specialist shops have survived the downward slide, and all sorts of ladies of the night, from old-hat sexagenarians to black beauties to Chinese cherries freshly settled in the capital, still line the street from Strazzy Saint-D to Chât-a-lay.

Les histoires farfelues racontées dans Paname Underground ne se déroulent pas en banlieue parisienne mais dans la capitale même, l'auteur ayant concocté un style distinct en se servant d'un grand nombre de voix différentes comme source d'inspiration. Pour traduire le premier chapitre de ce roman, je me suis appuyée sur les techniques les plus ludiques du parler jeune global, jouant encore plus avec les mots pour imprégner le texte d'un idiolecte particulier qui vient agrémenter le style relâché. Dans ma traduction, j'ai donc eu recours à des expressions inhabituelles (five in the AM) et à des structures parallèles (black beauties and Chinese cherries), en plus du travail sur le son, perceptible au niveau de la consonance (sight/'cept, few fatties, porn popped).

\section{Rhapsodie des oubliés}

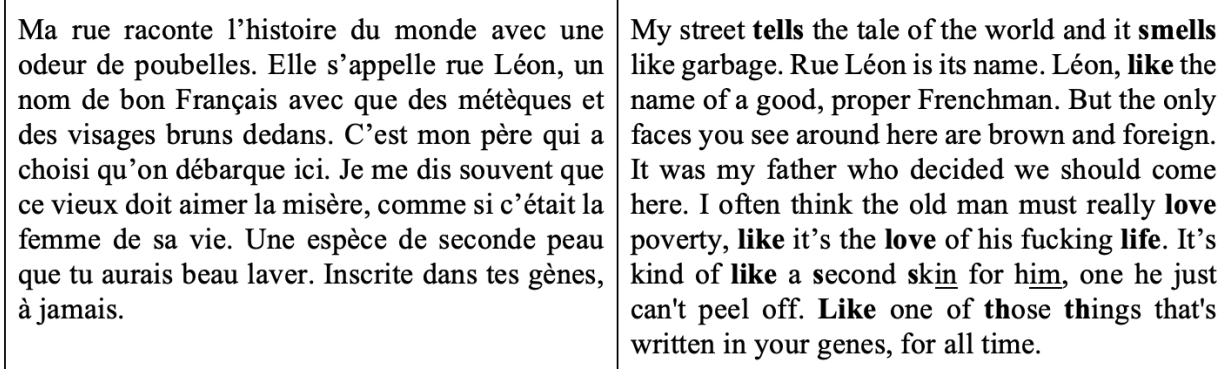

My street tells the tale of the world and it smells like garbage. Rue Léon is its name. Léon, like the name of a good, proper Frenchman. But the only faces you see around here are brown and foreign. It was my father who decided we should come here. I often think the old man must really love poverty, like it's the love of his fucking life. It's kind of like a second skin for him, one he just can't peel off. Like one of those things that's written in your genes, for all time.

Le dernier extrait que je commenterai dans cet article est tiré du premier chapitre du roman Rhapsodie des oubliés, dont l'histoire se déroule dans le 18e arrondissement de Paris. Pour traduire la voix du très jeune narrateur, Abad, qui a treize ans, $\mathrm{j}$ 'ai de nouveau eu recours à la répétition de sons, soulignés dans le tableau en gras, ainsi que l'accumulation, en reprenant les mots «like» et «love» plusieurs fois pour créer des échos à l'intérieur du passage. 


\section{Conclusion}

Face à l'impossibilité de prendre appui sur la langue pour ancrer un roman traduit dans un contexte géographique et social précis, on peut s'inspirer des techniques utilisées dans le rap pour développer une nouvelle stratégie de traduction qui évite de déplacer le texte dans un lieu différent et de trahir le choix des personnages d'utiliser une langue non standard. L'emploi de traits de la langue anglaise typiquement utilisés à l'oral par un nombre important de jeunes anglophones a servi de base pour cette stratégie, et le fait d'insérer dans des phrases souples les caractéristiques fondamentales du rap permet au traducteur d'intégrer dans la traduction un soupçon de résistance à la langue standard. Le «parler jeune global» tisse ainsi des liens implicites entre les fonctions ludiques et rebelles de l'argot et du rap, permettant au roman de satisfaire à son besoin de fonctionner comme un texte indépendant en anglais, apte à être diffusé à travers le monde anglophone.

\section{BIBLIOGRAPHIE}

Antoine, Fabrice (ed.). Argots, Langue familière et accents en traduction. Villeneuve d'Ascq :

Université Charles-de-Gaulle-Lille III, 2004.

Bradley, Adam. Book of Rhymes. New York: Basic Civatas Books, 2009.

Bradley, Adam et DuBois, Andrew (ed.). The Anthology of Rap. New Haven: Yale University Press, 2010.

Chang, Jeff. Can't Stop, Won't Stop: a History of the Hip-Hop Generation. New York: Picador, 2005.

Coleman, Julie (ed.). Global English Slang: Methodologies and Perspectives. New York: Routledge, 2014

Doran, Meredith. "Alternative French, Alternative Identities: Situating Language in la Banlieue." Contemporary French and Francophone Studies: 11:4 (2007): 497-508.

François-Geiger, Denise. "Panorama des argots contemporains.” Langue française : 90:1 (1991) : 5-9.

Mucchielli, Laurent. "Le rap : tentative d'expression politique de jeunes des quartiers relégués." Mouvements 3 (1999) : 60-66.

Partridge, Eric. Slang: To-day and Yesterday. London : George Routledge \& Sons, 1933.

Valdman, Albert. "La Langue des faubourgs et des banlieues : de l'argot au français populaire."

The French Review, 73 (2000): 1179-1192.

Corpus

Aouine, Sofia. Rhapsodie des oubliés. Paris : Éditions de la Martinière, 2019.

Goujon, Patrick. Moi non. Paris : Gallimard, 2003.

Lopez, David. Fief. Paris : Éditions du Seuil, 2017. 
Zarca. Paname Underground. Paris : Éditions Goutte d'Or, 2017.

\section{NOTES}

1. "[...] there are far more than those: the way you walk, the way you talk, the way you look, the way you communicate" - DJ Kool Herc

2. "Slang is informal, non-technical language that often seems novel to the user and/or listener, and that challenges a social or linguistic norm. It can also imply complicity in value judgements and thus play a performative role in defining personal or group identity."

\section{INDEX}

Thèmes : Dance

Mots-clés : rap, argot, roman urbain, jeunesse, flow

Keywords : rap, slang, urban novel, youth, flow

\section{AUTEURS}

\section{TIFFANE LEVICK}

Maître de conférences

Université Toulouse Jean-Jaurès

tiffane.levick@univ-tlse2.fr 